\title{
Correlations between plasma endothelin-I levels and breakthrough pain in patients with cancer
}

This article was published in the following Dove Press journal:

OncoTargets and Therapy

8 December 2015

Number of times this article has been viewed

\section{Xue-bin Yan \\ Tuo-chao Peng \\ Dong Huang}

Department of Anesthesiologist, The Third Xiangya Hospital of Central South University, Changsha, Hunan Province, People's Republic of China
Correspondence: Dong Huang Department of Anesthesiologist, The Third Xiangya Hospital of Central South University, 138 Tongzipo Road, Yuelu District, Changsha 4I00I3, Hunan Province, People's Republic of China Tel $+8673|886| 8 \mid 50$ Fax +8673 I 886I 8I5I Email donghuanghd@163.com
Abstract: Endothelin-1 (ET-1) may be involved in driving pain in patients with advanced cancer. However, a few studies focus on the role of ET-1 in breakthrough pain (BP). The aim of this pivotal study was to explore the correlation between the plasma (ET-1) level and BP intensity. A total of 40 patients were enrolled in the study, and they were divided into two groups: BP group and non-BP group. Moreover, 20 healthy adults were used as the normal control group. Pain intensity was measured using visual analog scale (VAS) scores of 1-10. Plasma ET-1 levels were detected by an ET radioimmunoassay kit. Subsequently, the correlation of ET-1 level with the VAS score and cancer types was analyzed by Pearson's correlation coefficient. The plasma ET-1 level in the BP group $(35.31 \pm 8.02 \mathrm{pg} / \mathrm{mL})$ was higher than that in the non-BP group $(29.51 \pm 6.78 \mathrm{pg} / \mathrm{mL})$ and the normal control group $(24.77 \pm 10.10 \mathrm{pg} / \mathrm{mL}$, $P<0.05)$. In addition, the VAS score in the BP group (7.45 \pm 0.82$)$ was higher than that in the non-BP group $(2.80 \pm 1.23, P<0.05)$. The plasma ET-1 level was positively correlated with the VAS score of the BP group (Pearson's $r=0.42$ ). There was no significant correlation between the plasma ET-1 level and VAS score of the non-BP group (Pearson's $r=-0.22$ ) or/and cancer types $(P>0.05)$. The elevated plasma ET-1 levels were positively related to $\mathrm{BP}$, and targeting ET-1 may provide a novel pain-reducing therapeutic treatment in BP.

Keywords: visual analog scale, correlation, cancer types, background pain

\section{Introduction}

Breakthrough pain (BP) is an acute transitory pain that occurs suddenly and breaks through the normal pain management. BP usually interrupts background pain that can be well controlled by medications. BP is generally divided into three types: idiopathic pain, incidental pain, and the end of dose pain. ${ }^{1}$ Moreover, the specific characteristics for further defining the BP include its predictability and relation to the fixed dose of opioid medication, temporal features, and precipitating events. ${ }^{2}$ The underlying mechanism of BP may be nociceptive, neuropathic, or mixed, ${ }^{3,4}$ and it may be also associated with the release of cytokines. ${ }^{5}$ However, the current knowledge seems to be insufficient.

Endothelin-1 (ET-1), a 21-residue vasoactive peptide, is produced by vascular endothelial cells ${ }^{6}$ and is a potent vasoconstrictor peptide that constricts blood vessels and raises blood pressure. ${ }^{7}$ Many reports on animal ${ }^{8}$ and human studies ${ }^{9}$ have indicated that ET-1 is involved in the pathogenesis of pain. Subcutaneous administration of ET-1 to a rat induces pain-like behavior and excites the nociceptive fibers selectively through the activation of $\mathrm{ET}_{\mathrm{A}}$ receptors. ${ }^{8,10}$ The levels of ET-1 are significantly increased in blister fluid in the complex regional pain syndrome type $1 .{ }^{11}$ ET-1 may be directly or indirectly involved in driving pain in patients with advanced cancer. ${ }^{12}$ However, there are a few studies ${ }^{13}$ that focus on the role of ET-1 in BP. 
The visual analog scale (VAS) score has been regarded as a valid and reliable tool for acute pain measurement. ${ }^{14}$ This pivotal study was designed to investigate the relationship between ET-1 level and BP intensity by the VAS score and the cancers types. The ET-1 level in patients with and without BP was detected. We are committed to reveal the role of ET-1 in the progression of BP and to discuss the possibility of applying ET-1 as a marker of BP diagnosis.

\section{Methods}

This pivotal study was approved by the Ethics Committee of The Third Xiangya Hospital of Central South University in accordance with the Declaration of Helsinki. Written informed consent was obtained from all the participants.

\section{Patients}

A total of 40 patients including 25 males and 15 females aged 30-70 years (mean age: $51.18 \pm 10.43$ years) were enrolled in the study from July 2011 to January 2012 at The Third Xiangya Hospital of Central South University, People's Republic of China. The cases included ten patients with rectal cancers, ten patients with liver cancers, two patients with splenic lymphomas, four patients with gastric cancers, eight patients with colon cancers, and six patients with breast cancers (Table 1). All the patients were divided into two groups: BP group and non-BP group. Additionally, 20 healthy adults who had the same ages with the patients in BP group were set as control group. Comparisons of general conditions (age and sex) among the three groups were conducted, and there was no significant difference in age and sex ratio among patients and controls (Table 1).

The selected patients must meet all the following criteria: 1) without obvious hypertension and heart and lung diseases; 2) without psychiatric history; 3) both the BP and non-BP groups had pathology clinical diagnoses; 4) BP diagnosis was performed according to the Science Committee of the Association for Palliative Medicine of Great Britain and Ireland; and 5) the age ranged from 30 years to 70 years.

Moreover, the patients with one or more of the following exclusion criteria were excluded: 1) patients who suffered from BP due to the insufficiency of last dosage, ie, BP occurred at 1-2 hours before the next administration; 2) patients with hypertension, coronary heart disease, severe arrhythmia, or with severe dysfunction in heart, liver, or kidney; 3) patients

Table I Normal clinical data in three groups $(\mathrm{n}=20, \bar{x} \pm s)$

\begin{tabular}{llll}
\hline Variable & BP group & Non-BP group & Normal control group \\
\hline Sex (M/F) & $12 / 8$ & $13 / 7$ & $\mathrm{II} / 9$ \\
Age (years) & $49.10 \pm 9.75$ & $53.25 \pm 10.92$ & $44.60 \pm 9.52$ \\
\hline
\end{tabular}

Abbreviations: BP, breakthrough pain; M, male; F, Female; s, standard deviation. recently treated for alcoholism or drug abuse; 4) patients with respiratory depression, anoxia, excessive bleeding, or severe shock during BP or treatment; 5) abnormal indexes because of reagents or equipment disorder; 6) patients who did not execute the protocol strictly, quitted the treatment, or died in the period of this study; 7) patients who did not coordinate with the doctors; and 8) patients with psychiatric history.

\section{Measurement of ET-I in plasma}

Before VAS assessing, $2 \mathrm{~mL}$ fasting venous blood was obtained from each patient and normal control after fasting overnight. Palliative chemo- and radiotherapy or surgery was performed in patients after sampling. Plasma was separated by centrifuging the blood sample at 2,000 rpm for 10 minutes and stored at $-80^{\circ} \mathrm{C}$. ET-1 levels in each sample were detected by an ET radioimmunoassay kit (Beijing North Institute of Biological Technology, Beijing, People's Republic of China) according to the manufacturer's instructions.

\section{BPVAS assessing}

Patients with chronic cancer pain or BP were treated with morphine hydrochloride sustained-release tablets (Southwest Pharmaceutical Co., Ltd., Chongqing, People's Republic of China) with an average dose of $180 \mathrm{mg} / \mathrm{d}$ for $>7$ days. Complications such as nausea, vomiting, and dizziness were cured during the treatment. Pain intensity was assessed using the VAS, and a ten-point categorical scale was recorded. The frequency of BP throughout the day and onset of relief were assessed. Patients who had the VAS score higher than 7 were considered to have severe pain, score 4-6 to have moderate pain, and score 1-3 to have mild pain. The clinical data, including frequentness, duration, intensity, inducement of pain, and the effect of treatment were recorded.

\section{Statistical analysis}

All data were expressed as mean \pm standard error of the mean. To determine the correlation between the ET-1 level and VAS score in the BP and non-BP groups, the data of the ET-1 level and VAS score were analyzed by Pearson's correlation coefficient. Statistical analysis was performed using $F$-test and Student's $t$-test by SPSS 18.0 software (SPSS Inc., Chicago, IL, USA). The value $P<0.05$ was considered statistically significant.

\section{Results}

\section{Correlation of the plasma ET-I level with pain intensity}

As shown in Table 2, the plasma ET-1 level in the BP group $(35.31 \pm 8.02 \mathrm{pg} / \mathrm{mL})$ was higher than that in the non-BP 
Table 2 The ET-I levels and VAS score in three groups

Parameter BP group Non-BP group Normal control group

ET-I $(\mathrm{pg} / \mathrm{mL}) \quad 35.31 \pm 8.02 \quad 29.51 \pm 6.78^{*} \quad 24.77 \pm 10.10^{* * \#}$

VAS score $\quad 7.45 \pm 0.82 \quad 2.80 \pm 1.23^{*} \quad-$

Notes: ${ }^{* P}<0.05$ vs $B P$ group; ${ }^{\# P<0.05}$ vs non-BP group.

Abbreviations: BP, breakthrough pain; ET-I, endothelin-I; VAS, visual analog scale.

group $(29.51 \pm 6.78 \mathrm{pg} / \mathrm{mL}, P<0.05)$ and the normal control group $(24.77 \pm 10.10 \mathrm{pg} / \mathrm{mL}, P<0.05)$. In addition, the plasma ET-1 level in the non-BP group was higher than that in the normal control group $(P<0.05)$. The VAS score in the BP group was higher than that in the non-BP group $(7.45 \pm 0.82$ vs $2.80 \pm 1.23, P<0.05)$.

The results of correlation analysis showed that the plasma ET-1 level was positively correlated with the VAS score in the BP group (Pearson's $r=0.42$ ). In addition, there was no significant correlation between the plasma ET-1 level and VAS score in the non-BP group (Pearson's $r=-0.22$ ).

\section{The ET-I levels andVAS score in different kinds of cancers}

No significant difference was observed between the plasma ET-1 level and VAS score of cancer types $(P>0.05)$.

\section{Discussion}

BP, a transitory exacerbation of pain superimposed on a background pain, has been reported in $\sim 50 \%-75 \%$ of patients with cancer. ${ }^{15}$ In this study, we found that the plasma ET-1 levels in patients with $\mathrm{BP}$ were higher than those in patients without $\mathrm{BP}$ and in normal controls $(P<0.05)$. We also demonstrated that the VAS score of patients with $\mathrm{BP}$ was higher than that of patients without $\mathrm{BP}(P<0.05)$. After performing the correlation analysis, we revealed that the plasma ET-1 level was positively correlated with the VAS score of the patients with BP (Pearson's $r=0.42$ ).

ET-1 has been reported to be involved in many aspects of cancer progression, such as promoting cell proliferation and tumor growth and inhibiting apoptosis. ${ }^{16,17}$ Overexpression of ET-1 has been found in many cancer types, including prostate cancer ${ }^{18}$ and colon cancer. ${ }^{19}$ The mechanism of ET-1 improving cancer-related pain may be like the following: ET-1 could directly activate nociceptors in human ${ }^{20}$ and rat. ${ }^{21}$ In addition, ET-1 enhances nociception induced by other algogens, including serotonin, formalin, and capsaicin. ${ }^{22}$

Previous studies have reported that the elevated plasma ET-1 levels were related to acute or subacute painful conditions. ${ }^{9,11}$ Mangiafico et $\mathrm{al}^{23}$ have revealed that the plasma ET-1 levels of patients with peripheral arterial occlusive disease were statistically higher than those of the normal controls, and they proposed that the elevated ET-1 levels could be a marker of ischemic acute endothelial damage. The same conditions have been found in other studies, such as patients with cardiac syndrome-X ${ }^{24}$ sickle cell disease, ${ }^{25}$ or complex regional pain syndrome. ${ }^{9}$ This may suggest that the elevated ET-1 levels could be found shortly after acute or subacute pain and are more likely to be presented in acute painful conditions, which is consistent with our study with higher ET-1 levels of the patients with BP compared to those of the patients without BP.

It has been shown that the concentration of ET-1 has a more direct correlation to induce pain levels in mice. ${ }^{26}$ A previous clinical study has demonstrated that a receptor of ET-1, $\mathrm{ET}_{\mathrm{A}}$ receptor antagonist, is promising in improving the cancer-related pain. ${ }^{18}$ In our study, we found that the VAS score in the patients with BP was higher than that in the patients without BP $(P<0.05)$. The plasma ET-1 levels were positively correlated with the VAS score in the BP group (Pearson's $r=0.42$ ). Overall, all these results suggest that targeting ET-1 may provide a novel pain-reducing therapeutic treatment for BP.

However, no significant difference was observed between the ET-1 levels and VAS score in different kinds of cancers $(P>0.05)$. This result may be due to the small sample size for every kind of cancer. As a result, further multivariate analysis (considering sex differences, course of disease, and setting cares) with larger sample size is needed to confirm our results.

\section{Limitations}

However, there are some potential limitations existing in the current survey. First, it is absent of a standard for assessment of BP. Second, the impact factors of BP such as activity, mood, work, and social relations were not calculated. In addition, more mechanisms involving ET-1 in BP are still needed to be explored in further studies.

\section{Conclusion}

The current study demonstrated that the elevated plasma ET-1 levels were related to BP intensity in patients with cancer, and targeting ET-1 may provide a novel pain-reducing therapeutic treatment for cancer patients with BP.

\section{Acknowledgment}

This study was supported by the National Natural Science Foundation of People's Republic of China (Grant No 81172546).

\section{Disclosure}

The authors report no conflicts of interest in this work. 


\section{References}

1. Davis MP, Walsh D. Epidemiology of cancer pain and factors influencing poor pain control. Am J Hosp Palliat Care. 2004;21(2):137-142.

2. Mishra S, Bhatnagar S, Chaudhary P, Rana SP. Breakthrough cancer pain: review of prevalence, characteristics and management. Indian $J$ Palliat Care. 2009;15(1):14.

3. Hwang SS, Chang VT, Kasimis B. Cancer breakthrough pain characteristics and responses to treatment at a VA medical center. Pain. 2003; 101(1):55-64.

4. Caraceni A, Portenoy RK. An international survey of cancer pain characteristics and syndromes. Pain. 1999;82(3):263-274.

5. Svendsen KB, Andersen S, Arnason S, et al. Breakthrough pain in malignant and non-malignant diseases: a review of prevalence, characteristics and mechanisms. Eur J Pain. 2005;9(2):195-206.

6. Xu D, Emoto N, Giaid A, et al. ECE-1: a membrane-bound metalloprotease that catalyzes the proteolytic activation of big endothelin-1. Cell. 1994;78(3):473-485.

7. Yanagisawa M, Kurihara H, Kimura S, et al. A novel potent vasoconstrictor peptide produced by vascular endothelial cells. Nature. 1988; 332(6163):411-415.

8. Gokin AP, Fareed MU, Pan HL, Hans G, Strichartz GR, Davar G. Local injection of endothelin-1 produces pain-like behavior and excitation of nociceptors in rats. J Neurosci. 2001;21(14):5358-5366.

9. Eisenberg E, Erlich T, Zinder O, et al. Plasma endothelin-1 levels in patients with complex regional pain syndrome. Eur J Pain. 2004;8(6): 533-538.

10. Davar G, Hans G, Fareed MU, Sinnott C, Strichartz G. Behavioral signs of acute pain produced by application of endothelin-1 to rat sciatic nerve. Neuroreport. 1998;9(10):2279-2283.

11. Groeneweg JG, Huygen FJ, Heijmans-Antonissen C, Niehof S, Zijlstra FJ. Increased endothelin-1 and diminished nitric oxide levels in blister fluids of patients with intermediate cold type complex regional pain syndrome type 1. BMC Musculoskelet Disord. 2006;7(1):91.

12. Davar G. Endothelin-1 and metastatic cancer pain. Pain Med. 2001; 2(1):24-27.

13. Urch C. The pathophysiology of cancer-induced bone pain: current understanding. Palliative Med. 2004;18(4):267-274.
14. Kelly AM. Does the clinically significant difference in visual analog scale pain scores vary with gender, age, or cause of pain? Acad Emerg Med. 1998;5(11):1086-1090.

15. Gómez-Batiste X, Madrid F, Moreno F, et al. Breakthrough cancer pain: prevalence and characteristics in patients in Catalonia, Spain. $J$ Pain Symptom Manage. 2002;24(1):45-52.

16. Nelson JB, Udan MS, Guruli G, Pflug BR. Endothelin-1 inhibits apoptosis in prostate cancer. Neoplasia. 2005;7(7):631-637.

17. Rosanò L, Spinella F, Di Castro V, et al. Endothelin-1 is required during epithelial to mesenchymal transition in ovarian cancer progression. Exp Biol Med. 2006;231(6):1128-1131.

18. Lalich M, McNeel DG, Wilding G, Liu G. Endothelin receptor antagonists in cancer therapy. Cancer Invest. 2007;25(8):785-794.

19. Asham E, Shankar A, Loizidou M, et al. Increased endothelin-1 in colorectal cancer and reduction of tumour growth by ET(A) receptor antagonism. Br J Cancer. 2001;85(11):1759.

20. Namer B, Hilliges M, Orstavik K, et al. Endothelin1 activates and sensitizes human C-nociceptors. Pain. 2008;137(1):41-49.

21. Joseph EK, Levine JD. Sexual dimorphism in endothelin-1 induced mechanical hyperalgesia in the rat. Exp Neurol. 2012;233(1):505-512.

22. Piovezan AP, D'Orleans-Juste P, Tonussi C, Rae GA. Endothelins potentiate formalin-induced nociception and paw edema in mice. Can J Physiol Pharmacol. 1997;75(6):596-600.

23. Mangiafico RA, Malatino LS, Spada RS, et al. Treadmill exerciseinduced release of endothelin-1 in patients with peripheral arterial occlusive disease at Fontaine stage IIb. Int Angiol. 2000;19(1):14-17.

24. Michelakakis N, Petropoulou E, Lazaros G, et al. Comparison of endothelin-1 levels at rest and during exercise between patients with cardiac syndrome-X and healthy people. Acta Cardiol. 1997;53(1):3-6.

25. Graido-Gonzalez E, Doherty JC, Bergreen EW, Organ G, Telfer M, McMillen MA. Plasma endothelin-1, cytokine, and prostaglandin E2 levels in sickle cell disease and acute vaso-occlusive sickle crisis. Blood. 1998;92(7):2551-2555.

26. Pickering V, Gupta RJ, Quang P, Jordan RC, Schmidt BL. Effect of peripheral endothelin-1 concentration on carcinoma-induced pain in mice. Eur J Pain. 2008;12(3):293-300.
OncoTargets and Therapy

\section{Publish your work in this journal}

OncoTargets and Therapy is an international, peer-reviewed, open access journal focusing on the pathological basis of all cancers, potential targets for therapy and treatment protocols employed to improve the management of cancer patients. The journal also focuses on the impact of management programs and new therapeutic agents and protocols on

\section{Dovepress}

patient perspectives such as quality of life, adherence and satisfaction The manuscript management system is completely online and includes a very quick and fair peer-review system, which is all easy to use. Visit http://www.dovepress.com/testimonials.php to read real quotes from published authors. 